\title{
Liraglutide exerts a bone-protective effect in ovariectomized rats with streptozotocin-induced diabetes by inhibiting osteoclastogenesis
}

\author{
BINHONG WEN, LU ZHAO, HONGMEI ZHAO and XIAOCHEN WANG \\ Department of Endocrinology, The People's Hospital of China Medical University, \\ The People's Hospital of Liaoning Province, Shenyang, Liaoning 110016, P.R. China
}

Received July 31, 2017; Accepted March 7, 2018

DOI: $10.3892 /$ etm.2018.6043

\begin{abstract}
Liraglutide, a glucagon-like peptide-1 receptor agonist, is an anti-diabetic medicine associated with a reduced risk of fracture in diabetic patients. In the present study, rats with streptozotocin (STZ)-induced diabetes and/or bilateral ovariectomy (OVX) were treated with liraglutide for eight weeks. Liraglutide treatment increased insulin secretion and managed blood glucose levels in the rats following STZ-induced diabetes. In addition, STZ- and OVX-induced reduction of femoral bone mineral density and destruction of bone microarchitecture were alleviated by liraglutide. STZ decreased, whereas OVX increased, serum osteocalcin (OC) level (a bone formation marker) and osteoblast counts in the trabecular bone. OVX, however not STZ, markedly increased the level of serum c-terminal telopeptide of type 1 collagen (CTX-1, a bone resorption marker) and osteoclast counts in the trabecular area. Liraglutide treatment significantly increased serum OC levels in all three osteoporotic models, however had minimal effects on osteoblast counts. Furthermore, liraglutide significantly decreased serum CTX-1 level and osteoclast numbers in OVX and STZ+OVX rats. Furthermore, the present study examined the mRNA expression and serum concentrations of osteoprotegerin (OPG) and receptor activator of nuclear factor- $\mathrm{kB}$ ligand (RANKL), and liraglutide significantly decreased the RANKL/OPG ratio compared with the untreated rats, indicating that osteoclastogenesis was inhibited by liraglutide. In summary, the results suggested that liraglutide ameliorates STZ+OVX-induced bone deterioration in the rat model, primarily through the inhibition
\end{abstract}

Correspondence to: Dr Binhong Wen, Department of Endocrinology, The People's Hospital of China Medical University, The People's Hospital of Liaoning Province, 33 Wenyi Road, Shenyang, Liaoning 110016, P.R. China

E-mail: bh177024@sina.com

Key words: diabetes, liraglutide, osteoclastogenesis, osteoporosis, ovariectomy of osteoclastogenesis. These preliminary findings propose a potentially beneficial effect of liraglutide on the bone health of postmenopausal diabetic patients.

\section{Introduction}

Diabetes mellitus is characterized by hyperglycemia and a serious of chronic complications, and it is a common metabolic disorder that occurs as a result of insulin deficiency or insulin resistance. Clinical evidences showed that bone mineral density (BMD) was below normal in patients with type 1 diabetes mellitus, a subtype of diabetes caused by inability of pancreatic beta-cells to secrete insulin (1). In addition, the risk of fracture in those with type 1 diabetes was 6.3- to 6.9-fold higher than that in age-matched control subjects, and the risk of fracture was increased to a less extent (1.4- to 1.7-fold) in those with type 2 diabetes (2). Diabetes-related decrease in BMD and weakened bone structure, of complicated pathophysiology, is considered to be one of the major factors that lead to bone fragility and elevated incidence of fractures in diabetic patients (1). Furthermore, some anti-diabetic drugs (like thiazolidinediones) could also directly affect bone metabolism and increase the risk of fracture (3).

Osteoporosis is a metabolic bone disease characterized by loss of bone mass and deterioration of bone microarchitecture. It mainly affects the elderly, especially postmenopausal women, and results in hip and vertebral fractures (4). Bone remodeling, referring to continuous cycles of bone resorption by osteoclasts and bone formation by osteoblasts, is in a homeostatic state under physiological conditions (5). In postmenopausal osteoporosis, estrogen deficiency leads to defective bone formation and strong bone resorption, accounting for progressive bone loss and an increased risk of fracture (5). Diabetic postmenopausal women, who experience a high risk of fracture, have been paid special attention to for the management of their bone health and prevention of fractures (6-9).

Liraglutide, a glucagon-like peptide-1 (GLP-1) receptor agonist (GLP-1 RA), is a novel anti-diabetic drug that mimics the endogenous GLP-1 to potentiate insulin secretion (10). A meta-analysis of fracture incidence in diabetic patients shows a reduced risk of incident fractures in the patients receiving liraglutide treatment (11). In addition, long-term administration of 
liraglutide has an anabolic bone effect in weight-reduced obese women (12). Further studies on animal models demonstrated that liraglutide could prevent bone loss and counter rapid bone deterioration in diabetic rodents $(13,14)$. Moreover, liraglutide could improve bone mass and architecture in non-diabetic osteoporotic rodent models with ovariectomy (OVX) $(15,16)$. So far, the potential bone-protective effect of liraglutide on the postmenopausal diabetic population has not been assessed. In the present study, we explored the effects of liraglutide on the osteoporotic bones in a rat model with streptozotocin (STZ)-induced diabetes and bilateral OVX. Furthermore, we investigated the modulatory effects of liraglutide on the markers of bone turnover in the diabetic/OVX rats.

\section{Materials and methods}

Animal models and treatment. All the animal were housed under standard housing conditions (12-h light/dark cycle, temperature of $22-24^{\circ} \mathrm{C}$ and relative humidity of 50-60\%, with access to food and water ad libitum) in the animal facility center at our institute, and all procedures on the animals were approved by the Institutional Animal Care and Use Committee of The People's Hospital of Liaoning Province (Shenyang, China). Nine-week-old female Sprague Dawley rats were randomly divided into seven groups: Sham, STZ, STZ+L, OVX, OVX+L, STZ+OVX, and STZ+OVX+L. To induce diabetes, the animals were fasted overnight, and then received an intraperitoneal injection of STZ (Solarbio, Beijing, China; dissolved in $0.1 \mathrm{M}$ citrate buffer, $\mathrm{pH} 4.5$ ) at a dose of $60 \mathrm{mg} / \mathrm{kg}$ body weight. Those in the Sham, OVX and OVX+L groups received an equal volume of the solvent via the same administration route. Blood was sampled through the tail vein $72 \mathrm{~h}$ later, and the rats with blood glucose levels of $16.7 \mathrm{mM}$ and above were considered to be successful induction of the diabetic model. Thereafter, bilateral OVX was performed on the rats in the OVX, OVX+L, STZ+OVX, and STZ+OVX+L groups following the previously described two-incision procedures (17). The same procedures were performed on the rest animals except for the ligation and excision of ovaries. One week later, liraglutide (Novo Nordisk, Copenhagen, Demark) was given to the rats in the $\mathrm{STZ}+\mathrm{L}, \mathrm{OVX}+\mathrm{L}$ and $\mathrm{STZ}+\mathrm{OVX}+\mathrm{L}$ groups by subcutaneous injection at a dose of $0.6 \mathrm{mg} / \mathrm{kg}$ daily for a total of eight weeks, and an equal volume of saline was given daily by subcutaneous injection to the other rats during the same period. The rats were monitored every day and the rats' body weights were recorded every week during the course of the experiment. Eight weeks after STZ administration, 1-2 out of 10 STZ-treated rats became very lean and inactive, the rat was euthanized by overdose anesthesia once it was no longer taking food (humane endpoint). These rats were not included for the analyses.

Intraperitoneal glucose tolerance test (IPGTT). At the end of eight-week liraglutide treatment, rats were fasted for $18 \mathrm{~h}$. Blood was sampled through the tail vein prior to $(0 \mathrm{~min})$ as well as 15, 30, 60 and 120 min after an intraperitoneal injection of glucose solution ( $2 \mathrm{~g} / \mathrm{kg}$ body weight). A blood drop on a blood glucose test strip was read for glucose concentration by the Safe-Accu Angel Blood Glucose Monitoring System (Sannuo, Changsha, China).
Serum biochemistry. After eight-week liraglutide treatment, the rats were euthanized with overdose chloral hydrate, and blood was withdrawn from each rat via the inferior vena cava. ELISA was performed to determine the levels of insulin, osteocalcin (OC), c-terminal telopeptide of type 1 collagen (CTX-1), osteoprotegerin (OPG) and receptor activator of nuclear factor- $\kappa \mathrm{B}$ ligand (RANKL) proteins in the serum using the commercially available ELISA kits (OC: LSBio, Seattle, WA, USA; insulin, CTX-1, OPG and RANKL: USCN, Wuhan, China) according to the manufacturer's instructions. The ratio of serum RANKL/OPG was then calculated.

Determination of BMD. Prior to STZ administration, and four and eight weeks after liraglutide treatment, BMD was measured at the proximal femurs in vivo using a GE-Lunar dual energy X-ray absorptiometer (GE healthcare, Madison, WI, USA). Each femur was measure three times for an average value.

Hematoxylin and eosin $(H \& E)$ and TRAP staining. H\&E and TRAP staining were performed nine weeks after STZ administration and/or OVX procedure. The right femur of each rat was fixed in $10 \%$ neutral buffered formalin and decalcified in $10 \%$ ethylenediamine tetraacetic acid disodium (EDTA-2Na) (pH 7.2) for 30 days. The decalcified femurs were dehydrated, embedded in paraffin, and sectioned with a Leica RM2235 microtome (Germany). The tissue sections were stained with $\mathrm{H} \& \mathrm{E}$ following routine procedures, followed by microscopic examination (magnification, $\mathrm{x} 400$ ) for histopathological evaluation and osteoblast counts. In order to characterize osteoclasts, the bone sections were subjected to TRAP staining as previously described (18). Six random fields on each H\&E- or TRAP-stained sections were photographed at 400x, and osteoblast/osteoclast number and bone perimeter/area on each microscopic image were determined using Image Pro Plus 6.0 (Media Cybernetics, Rockville, MD, USA). The average number of osteoblasts per bone perimeter (Ob.N/B.Pm) and the number of osteoclasts per tissue area (Oc.N/T.Ar, $\mathrm{mm}^{-2}$ ) were reported according to the guidelines by the American Society for Bone and Mineral Research Nomenclature Committee (19).

Reverse transcription-quantitative polymerase chain reaction $(R T-q P C R)$. The expressions of $O P G$ and $R A N K L$ mRNAs in the proximal femur were measured by qRT-PCR. The proximal femur was pulverized in liquid nitrogen, and total RNA was extracted from the bone tissues using a Total RNA Fast Extraction kit (BioTeke, Beijing, China) according to the manufacturer's instructions. The extracted RNA was reverse transcribed into cDNA using Super M-MLV Reverse Transcriptase (BioTeke). RT-qPCR was performed in Exicycler ${ }^{\mathrm{TM}} 96$ Realtime Quantitative Thermal Block (BIONEER, Daejeon, Korea) using SYBR-Green Master Mix (Solarbio) and the following primers: $O P G$ forward, 5'-GAC CCCAGAGCGAAACACG-3', and reverse, 5'-GGCACAGCA AACCTGAAGAA-3'; RANKL forward, 5'-CATCGGGTT CCCATAAAG-3', and reverse, 5'-GAAGCAAATGTTGGC GTA-3'; ACTB forward, 5'-GGAGATTACTGCCCTGGCTCC TAGC-3', and reverse, 5'-GGCCGGACTCATCGTACTCCT GCTT-3'. The expression levels of OPG and RANKL mRNAs 
were normalized to the expression level of $A C T B$ mRNA, an internal reference gene encoding $\beta$-actin, and the values were expressed as relative values to the Sham group. The ratio of RANKL/OPG was calculated accordingly.

Statistical analysis. All the data are presented as means \pm SD. Statistical analysis was performed using GraphPad Prism 5.0 (GraphPad Software Inc., La Jolla, CA, USA). Comparisons between groups were conducted using one-way analysis of variance (ANOVA) followed by Bonferroni's post hoc test. $\mathrm{P}<0.05$ was considered to indicate a statistically significant difference.

\section{Results}

Liraglutide attenuated STZ-induced diabetic conditions in rats. One week after STZ administration and/or OVX, the rats were treated with liraglutide for eight weeks. As shown in Fig. 1A, rat body weight dropped over time following STZ administration, and liraglutide attenuated the weight loss in STZ-treated rats. In addition, rat body weight increased after OVX as compared with sham-operated rats. As a GLP-1 analog that can potentiate insulin secretion (10), liraglutide significantly increased serum insulin concentrations in STZ and STZ+OVX rats after eight week treatment (Fig. 1B), and it did not affect serum estradiol levels in rats with STZ-induced diabetes and/or OVX procedure (data not shown). Moreover, fasting blood glucose (Fig. 1C) and glucose tolerance (Fig. 1D) were measured, and the results confirmed the anti-hyperglycemic effect of liraglutide in STZ-induced diabetes.

Liraglutide increased BMD and modulated bone metabolism in rats with STZ-induced diabetes and OVX. Femoral BMD was measured before STZ administration and/or OVX procedure, as well as four and eight weeks after liraglutide treatment. As shown in Fig. 2A, administration of STZ led to gradual reduction of BMD in the femur. In contrast, OVX caused a sudden decline in BMD as an early response, and BMD was slightly increased in the OVX rats at a later adaptive stage. In STZ+OVX rats, BMD declined quickly and kept at the low level. Liraglutide treatment maintained BMD after STZ induction. Moreover, liraglutide significantly attenuated the reduction of BMD in the rats with OVX or OVX+STZ.

Next, we assessed the serum levels of OC and CTX-1, the markers of bone formation and resorption, respectively. As shown in Fig. 2B, STZ decreased the level of OC in the serum, whereas OVX increased serum OC concentration. Liraglutide significantly increased serum OC concentrations in all three osteoporotic models, demonstrating an anabolic bone effect of liraglutide. On the other hand, OVX markedly elevated the level of serum CTX-1, while STZ had little effect on serum CTX-1 though (Fig. 2C). Liraglutide significantly reduced the elevation of serum CTX-1 in OVX and STZ+OVX rats as compared with the corresponding counterparts without liraglutide treatment. These results demonstrated that liraglutide promoted bone formation and markedly inhibited bone resorption in the rats with OVX and STZ-induced diabetes.

Liraglutide prevented bone destruction and reduced osteoclast numbers in STZ+OVX rats. Histological changes in the proximal femur were examined with $\mathrm{H} \& \mathrm{E}$ staining. As shown in Fig. 3, STZ or OVX resulted in thinning of trabeculae and increased numbers and sizes of empty bone lacunae in the proximal femur, while STZ+OVX exaggerated the pathological alterations compared with either single treatment. Compared to STZ+OVX group, STZ+OVZ+L group showed significant increases in the thickness of trabeculae and total bone mass in the proximal femur.

Next, the numbers of osteoblasts and osteoclasts were counted based on H\&E- and TRAP-stained sections, respectively (Figs. 3 and 4). STZ decreased, while OVX and STZ+OVX increased, the number of osteoblasts in the trabecular bone (Fig. 3B). Liraglutide treatment, however, did not show any significant effect on the osteoblasts at week 9 in rats with STZ-induced diabetes and/or OVX. On the other hand, STZ slightly while OVX markedly increased the number of osteoclasts in the proximal femur, whereas liraglutide treatment significantly reduced osteoclast number in the rats received OVX or co-treated with STZ+OVX (Fig. 4B). These data suggest that liraglutide may preserve bone mass and architecture in the OVX+STZ rats mainly by inhibiting osteoclast-mediated bone resorption.

Liraglutide decreased the $R A N K L / O P G$ ratio in osteoporotic rats. RANKL is an essential factor that drives osteoclast differentiation and activation (20), and OPG is a natural antagonist of RANKL (21). Hence, the RANKL/OPG ratio determines the commitment to osteoclast differentiation. The expression of $O P G$ and $R A N K L$ mRNAs in the femoral tissue was assessed by RT-qPCR. Compared with the sham-operated animals, either STZ or OVX significantly reduced the expression of $O P G$ mRNA in the femur, and OVX markedly increased femoral expression of RANKL mRNA (Fig. 5A and B). Liraglutide increased $O P G$ expression and decreased $R A N K L$ expression in the osteoporotic rat models as compared with the untreated counterparts. Moreover, STZ and OVX led to increased ratios of $R A N K L / O P G$ mRNA in the femur, which favored osteoclast differentiation. Liraglutide treatment, by contrast, significantly decreased the $R A N K L / O P G$ ratio as compared to the corresponding untreated groups (Fig. 5C). Consistently, STZ and/or OVX significantly decreased serum OPG level and increased serum RANKL level, resulting in increased ratios of serum RANKL/OPG (Fig. 5D-F). Liraglutide reversed the alterations in serum OPG and RANKL levels, and reduced the serum RANKL/OPG ratio in the osteoporotic models. Thus, these results indicated that STZ- and OVX-induced osteoclastogenesis was inhibited by liraglutide treatment.

\section{Discussion}

GLP-1 is an incretin hormone that is synthesized and secreted by gut L cells in response to food intake, and it stimulates insulin release by pancreatic beta-cells and suppresses glucagon secretion from alpha-cells (22). As GLP-1 is rapidly degraded in circulation, stable GLP-1 RAs are developed as a new class of anti-diabetic medications that mimic incretin activities in the body (10). Since expression of functional GLP-1 receptors was identified on osteoblasts (23), the potential beneficial effects of GLP-1 RAs has been evaluated experimentally on diabetic and non-diabetic models $(11,13,15,16,24,25)$. Previous studies have 

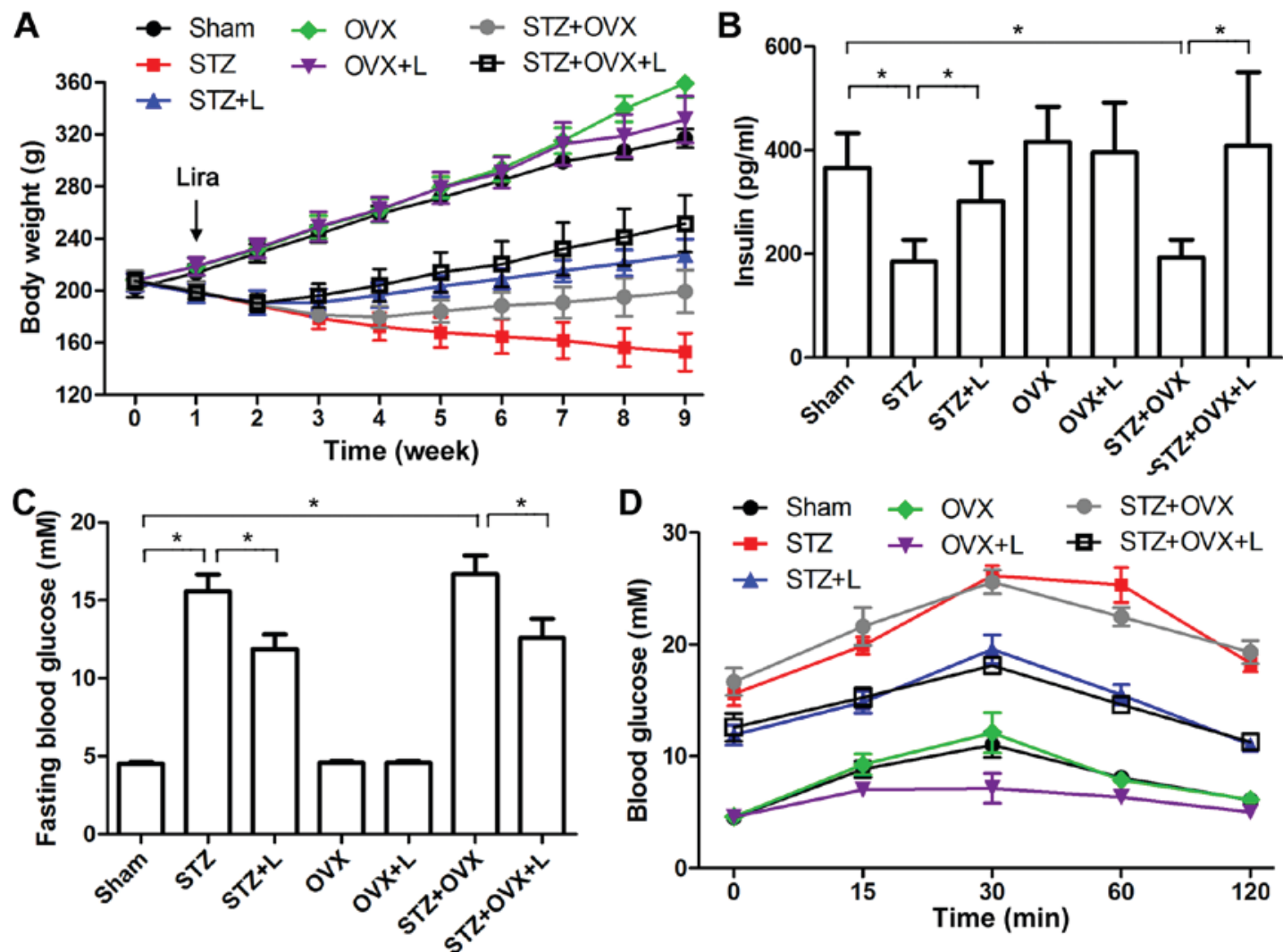

Figure 1. Body weight, serum insulin level and glucose tolerance in rats with and without liraglutide treatment following STZ-induced diabetes and/or OVX procedure. One week after STZ administration and/or OVX procedure, the rats were treated with liraglutide for eight weeks. (A) Rats' body weights were monitored every week. (B) The concentrations of serum insulin was determined by ELISA at the end of the liraglutide treatment. (C and D) After eight-week liraglutide treatment, the rats were fasted overnight. (C) The fasting blood glucose levels were measured, and (D) glucose tolerance was evaluated by IPGTT. The data are expressed as means $\pm \mathrm{SD}$ ( $\mathrm{n}=6$ per group). ${ }^{\mathrm{P}}<0.05$ indicates a significant difference between two groups. STZ, streptozotocin; OVX, ovariectomy.
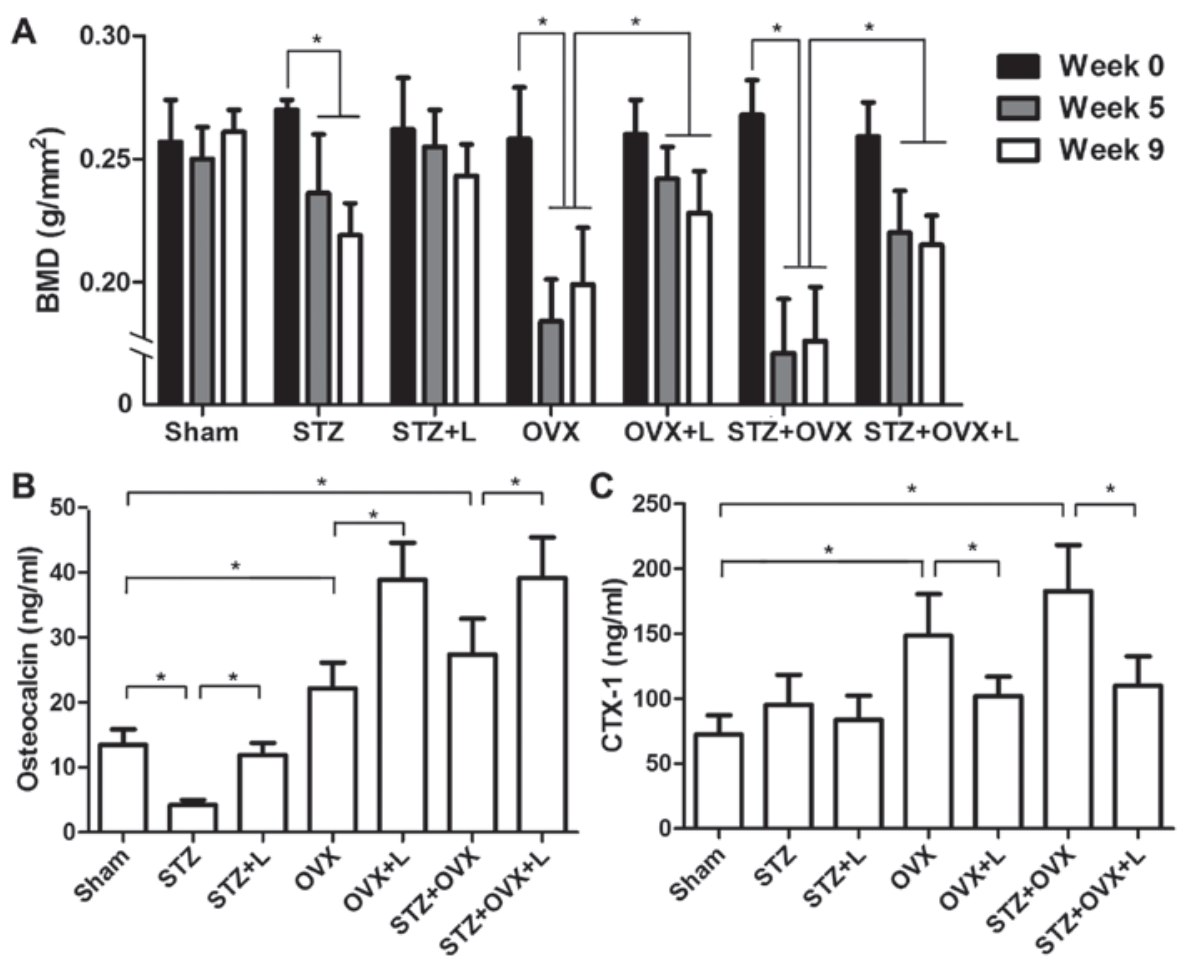

Figure 2. Effects of liraglutide treatment on BMD, osteocalcin and CTX-1 in rats with STZ induction and OVX. Administration of liraglutide started one week after STZ induction and/or OVX operation. (A) BMD was measured prior to, five and nine weeks after STZ administration and OVX. The serum levels of (B) osteocalcin and (C) CTX-1 were determined after eight-week liraglutide treatment. The data are expressed as means \pm SD ( $\mathrm{n}=6$ per group). " $\mathrm{P}<0.05$ indicates a significant difference between two groups. STZ, streptozotocin; OVX, ovariectomy; BMD, bone mineral density; CTX-1, c-terminal telopeptide of type 1 collagen 

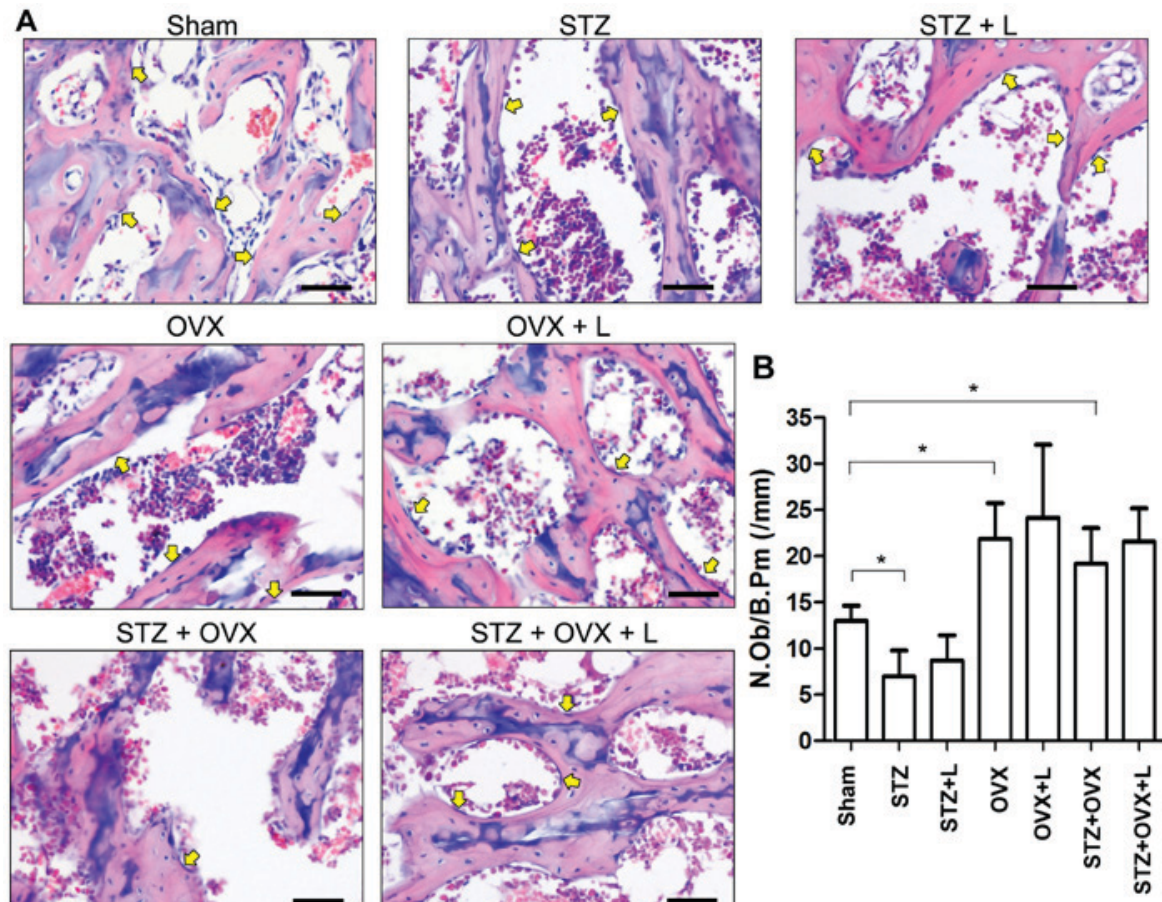

Figure 3. Histological evaluation of the proximal femur and osteoblast counts in osteoporotic rats with or without liraglutide treatment. (A) Nine weeks after STZ administration and OVX procedure, the proximal femurs of rats with and without liraglutide treatment were subjected to H\&E staining (scale $=50 \mu \mathrm{m}$ ). Yellow arrows point at osteoblasts. (B) The number of osteoblasts on each H\&E-stained section was counted, and N.Ob/B.Pm (/mm) was determined using the Image Pro Plus 6.0 analytic software. The data are expressed as means $\pm \mathrm{SD}$ ( $\mathrm{n}=6$ per group). ${ }^{*} \mathrm{P}<0.05$ indicates a significant difference. $\mathrm{STZ}$, streptozotocin; OVX, ovariectomy.
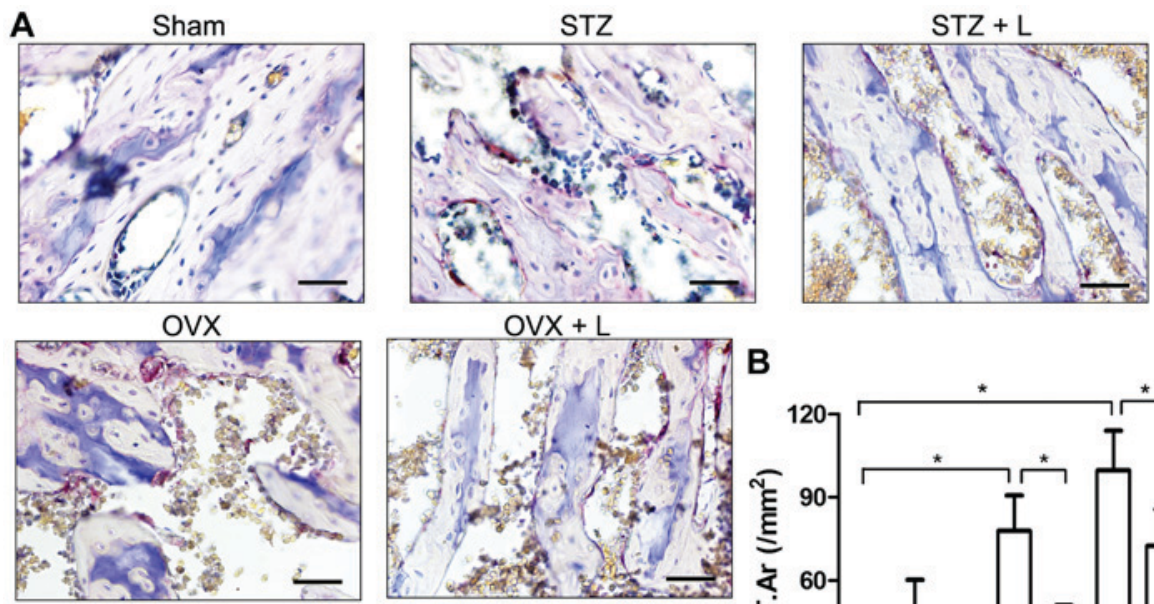

$\mathrm{STZ}+\mathrm{OVX}$
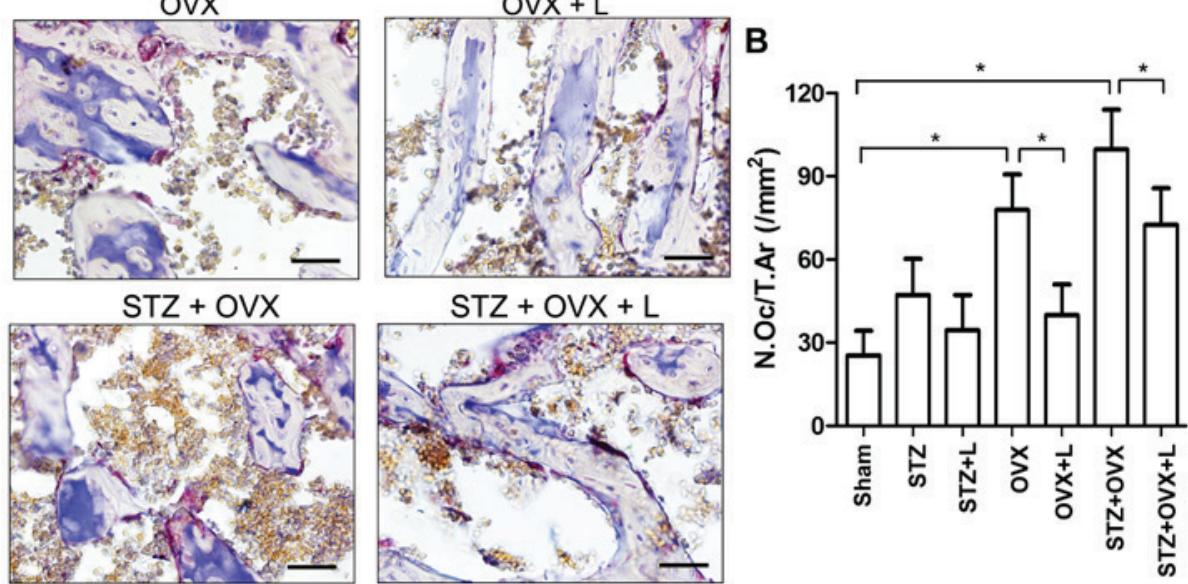

Figure 4. Evaluation of osteoclasts in the proximal femur of osteoporotic rats with or without liraglutide treatment. (A) Nine weeks after STZ administration and OVX procedure, the proximal femurs of rats with and without liraglutide treatment were subjected to TRAP staining (scale $=50 \mu \mathrm{m})$. Osteoclasts were stained in dark ruby. (B) The numbers of osteoclasts were counted based on TRAP-stained sections, and N.OC/T.Ar (/mm²) was determined by Image Pro Plus 6.0. The data are expressed as means $\pm \mathrm{SD}$ ( $\mathrm{n}=6$ per group). ${ }^{*} \mathrm{P}<0.05$ indicates a significant difference. $\mathrm{STZ}$, streptozotocin; OVX, ovariectomy.

demonstrated that liraglutide treatment could increase BMD, improve bone micro-architecture and restore mechanical properties of the bone in diabetic rodents $(13,14)$, and it also exerts beneficial effects on the bone in non-diabetic osteoporotic models $(15,16)$. In the present study, we constructed a rat model of osteoporosis induced by both diabetes and OVX, 

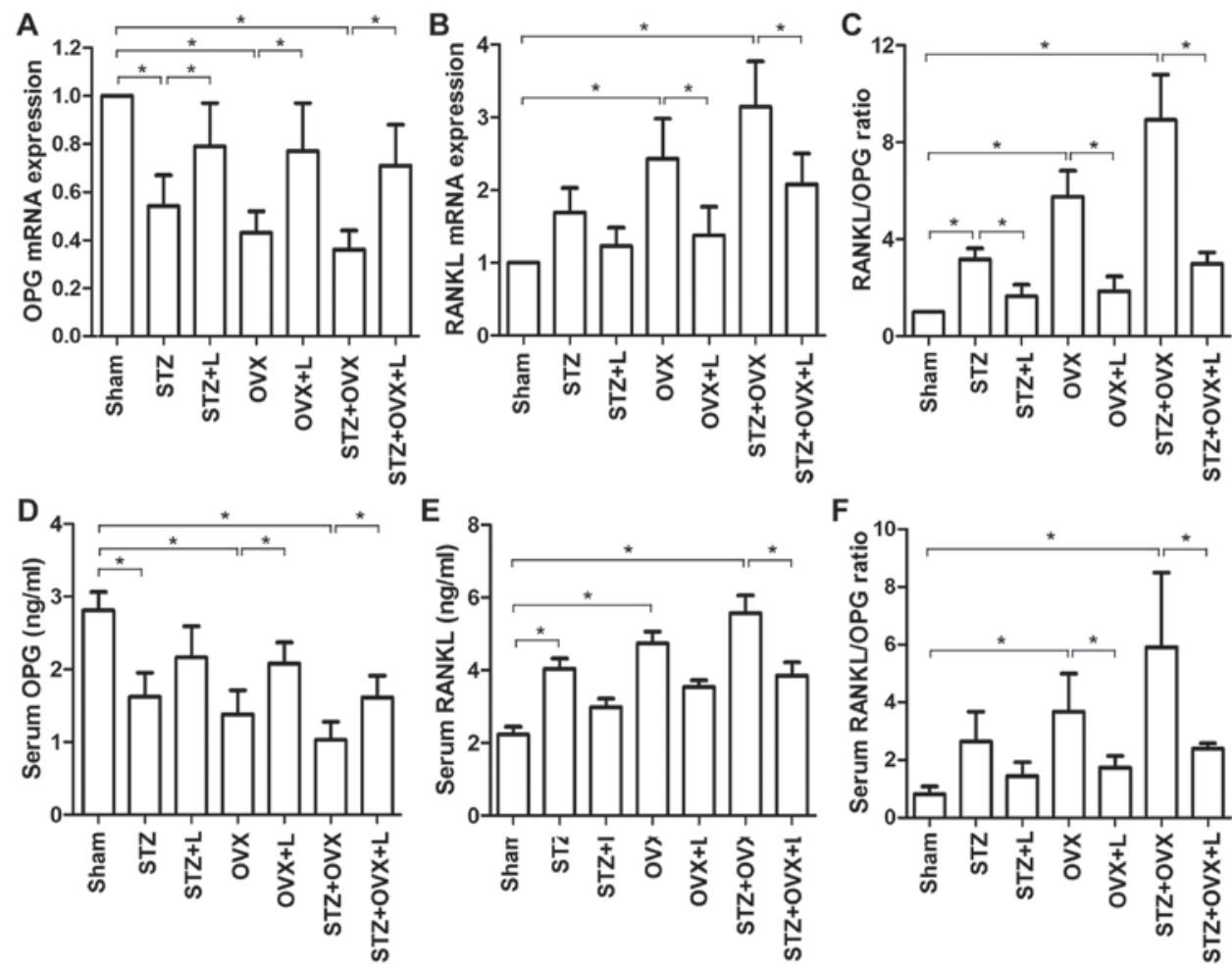

Figure 5. Assessment of RANKL/OPG ratio in the proximal femur and serum of osteoporotic rats with or without liraglutide treatment. Relative expression levels of (A) $O P G$ mRNA and (B) RANKL mRNA in the proximal femur were determined by RT-qPCR and normalized to the Sham group. (C) The RANKL/OPG mRNA ratio in the proximal femur was calculated based on the RT-qPCR results. The concentrations of (D) OPG and (E) RANKL proteins in the serum were measured by ELISA, and $(\mathrm{F})$ the serum RANKL/OPG ratio was calculated accordingly. The data are expressed as means \pm SD ( $\mathrm{n}=6$ per group). ${ }^{*} \mathrm{P}<0.05$ indicates a significant difference. RANKL, receptor activator of nuclear factor- $\mathrm{kB}$ ligand; OPG; osteoprotegerin.

and showed that long-term liraglutide treatment was able to ameliorate the defects in the bone of the severely osteoporotic rats. Our study supports the bone-protective effect of liraglutide, and provides preliminary evidence for the potential benefits of liraglutide on the bone health of postmenopausal diabetic patients.

The bone is a metabolically active tissue that undergoes constant turnover through bone formation and resorption. In the present study, although both STZ and OVX led to bone loss in rats, their action mechanisms are apparently different. In the diabetic rats, serum OC and osteoblast count were markedly reduced, and these observations are consistent with previous reports $(13,26)$. In the meanwhile, serum CTX-1 and osteoclast count were only slightly increased in STZ-induced diabetes. These data imply that STZ-induced osteopenia was mainly attributed to impaired bone formation. In the OVX rats, by contrast, serum markers of bone formation (OC) and bone resorption (CTX-1) were both elevated, while both osteoblasts and osteoclasts were increased. These findings, in line with previous studies $(27,28)$, suggest that OVX-induced bone loss was due to an increase rate of bone turnover. Liraglutide was implicated to have an anabolic bone effect because it increased the bone formation marker (N-terminal propeptide of type 1 procollagen) in weight loss-induced bone mass reduction (12). This is similar with the phenomenon observed in our study that liraglutide treatment led to increased serum OC levels in STZ, OVX and STZ+OVX rats. Liraglutide and other GLP-1 RA have been demonstrated to exert osteogenic actions in animal models $(14,25)$. In a spontaneous diabetic rat model, liraglutide stimulates the expression of Runx2, a transcription factor that drives the expression of osteoblast-specific genes, as well as a number of bone formation markers such as alkaline phosphatase, collagen 1 and OC in the bone (14). Intriguingly, liraglutide elevated the level of bone formation marker but showed no effect on osteoblast counts in rats with STZ- and OVX-induced osteoporosis. Our results suggest that liraglutide promotes bone formation in the rats with STZand OVX-induced osteoporosis probably by enhancing the expression osteoblast-specific genes rather than promoting the proliferation or differentiation of osteoblasts.

In the STZ+OVX rats with high rates of bone formation and resorption, liraglutide treatment significantly reduced serum CTX-1 and osteoclast number, suggesting that liraglutide inhibited osteoclastogenesis and consequently attenuated bone resorption in the osteoporotic rats. It was reported that mice with GLP-1 receptor deficiency exhibited osteopenia, increased osteoclast numbers and elevated bone resorption (29), confirming an inhibitory role of GLP-1 signaling in osteoclastogenesis. During osteoclastogenesis, RANKL drives osteoclast differentiation (20) while OPG antagonizes RANKL action (21), and the RANKL/OPG ratio determines osteoclastogenesis in the bone. Here, we showed that both STZ and OVX increased RANKL/OPG ratio, which is consistent with previous studies (30-33). Liraglutide significantly reduced RANKL/OPG ratio compared to the untreated counterparts, indicating that liraglutide can inhibit osteoclastogenesis in STZ, OVX and STZ+OVX rats. GLP-1 and GLP-1 RA exendin-4 have been shown to reverse hyperlipidic-related 
osteopenia and decrease tibia RANKL/OPG ratio (24). Our data are consistent with the previous findings and suggest that liraglutide or GLP-1 signaling may suppress osteoclastogenesis via modulating the RANKL/OPG ratio.

In conclusion, the present study demonstrates a bone-preserving effect of liraglutide in a rat osteoporotic model with STZ-induced diabetes and OVX, and this effect is mainly associated with liraglutide-mediated suppression of osteoclastogenesis. Our data suggest a potential value of liraglutide in protecting the bone health of postmenopausal diabetic population, yet this idea needs to be assessed in future clinical studies.

\section{Acknowledgements}

This study was supported by grants from the Natural Science Foundation of Liaoning Province (no. 2015020658) and the Diagnostic and Therapeutic Capability Construction Project for Key Clinical Departments of Liaoning Provincial Hospital Reform (no. LNCCC-D35-2015).

\section{References}

1. Vestergaard P: Discrepancies in bone mineral density and fracture risk in patients with type 1 and type 2 diabetes-a meta-analysis. Osteoporos Int 18: 427-444, 2007.

2. Janghorbani M, Van Dam RM, Willett WC and Hu FB: Systematic review of type 1 and type 2 diabetes mellitus and risk of fracture. Am J Epidemiol 166: 495-505, 2007.

3. Grey A: Skeletal consequences of thiazolidinedione therapy. Osteoporos Int 19: 129-137, 2008.

4. Rachner TD, Khosla S and Hofbauer LC: Osteoporosis: Now and the future. Lancet 377: 1276-1287, 2011.

5. Zaidi M: Skeletal remodeling in health and disease. Nat Med 13: 791-801, 2007

6. Berberoglu Z, Yazici AC and Demirag NG: Effects of rosiglitazone on bone mineral density and remodelling parameters in Postmenopausal diabetic women: A 2-year follow-up study. Clin Endocrinol (Oxf) 73: 305-312, 2010.

7. Heilmeier U, Carpenter DR, Patsch JM, Harnish R, Joseph GB, Burghardt AJ, Baum T, Schwartz AV, Lang TF and Link TM: Volumetric femoral BMD, bone geometry and serum sclerostin levels differ between type 2 diabetic postmenopausal women with and without fragility fractures. Osteoporos Int 26: 1283-1293, 2015.

8. Henriksen DB, Alexandersen P, Hartmann B, Adrian CL, Byrjalsen I, Bone HG, Holst JJ and Christiansen C: Four-month treatment with GLP-2 significantly increases hip BMD: A randomized, placebo-controlled, dose-ranging study in postmenopausal women with low BMD. Bone 45: 833-842, 2009.

9. Nicodemus KK and Folsom AR; Iowa Women's Health Study: Type 1 and type 2 diabetes and incident hip fractures in postmenopausal women. Diabetes Care 24: 1192-1197, 2001.

10. Nauck MA: Incretin-based therapies for type 2 diabetes mellitus: Properties, functions and clinical implications. Am J Med 124 (Suppl 1): S3-S18, 2011.

11. Su B, Sheng H, Zhang M, Bu L, Yang P, Li L, Li F, Sheng C, Han Y, Qu S and Wang J: Risk of bone fractures associated with glucagon-like peptide-1 receptor agonists' treatment: A meta-analysis of randomized controlled trials. Endocrine 48: 107-115, 2015.

12. Iepsen EW, Lundgren JR, Hartmann B, Pedersen O, Hansen T, Jørgensen NR, Jensen JE, Holst JJ, Madsbad S and Torekov SS: GLP-1 receptor agonist treatment increases bone formation and prevents bone loss in weight-reduced obese women. J Clin Endocrinol Metab 100: 2909-2917, 2015.

13. Mansur SA, Mieczkowska A, Bouvard B, Flatt PR, Chappard D, Irwin $\mathrm{N}$ and Mabilleau G: Stable incretin mimetics counter rapid deterioration of bone quality in type 1 diabetes mellitus. J Cell Physiol 230: 3009-3018, 2015.

14. Sun HX, Lu N, Luo X, Zhao L and Liu JM: Liraglutide, the glucagon-like peptide-1 receptor agonist, has anabolic bone effects in diabetic Goto-Kakizaki rats. J Diabetes 7: 584-588, 2015.
15. Lu N, Sun H, Yu J, Wang X, Liu D, Zhao L, Sun L, Zhao H, Tao B and Liu J: Glucagon-like peptide-1 receptor agonist Liraglutide has anabolic bone effects in ovariectomized rats without diabetes. PLoS One 10: e0132744, 2015.

16. Pereira M, Jeyabalan J, Jorgensen CS, Hopkinson M, Al-Jazzar A, Roux JP, Chavassieux P, Orriss IR, Cleasby ME and Chenu C: Chronic administration of Glucagon-like peptide-1 receptor agonists improves trabecular bone mass and architecture in ovariectomised mice. Bone 81: 459-467, 2015.

17. Lasota A and Danowska-Klonowska D: Experimental osteoporosis-different methods of ovariectomy in female white rats. Rocz Akad Med Bialymst 49 (Suppl 1): S129-S131, 2004.

18. Moon YJ, Yun CY, Choi H, Ka SO, Kim JR, Park BH and Cho ES: Smad4 controls bone homeostasis through regulation of osteoblast/osteocyte viability. Exp Mol Med 48: e256, 2016.

19. Dempster DW, Compston JE, Drezner MK, Glorieux FH, Kanis JA, Malluche H, Meunier PJ, Ott SM, Recker RR and Parfitt AM: Standardized nomenclature, symbols and units for bone histomorphometry: A 2012 update of the report of the ASBMR histomorphometry nomenclature committee. J Bone Miner Res 28: 2-17, 2013.

20. Kong YY, Yoshida H, Sarosi I, Tan HL, Timms E, Capparelli C, Morony S, Oliveira-dos-Santos AJ, Van G, Itie A, et al: OPGL is a key regulator of osteoclastogenesis, lymphocyte development and lymph-node organogenesis. Nature 397: 315-323, 1999.

21. Simonet WS, Lacey DL, Dunstan CR, Kelley M, Chang MS, Luthy R, Nguyen HQ, Wooden S, Bennett L, Boone T, et al: Osteoprotegerin: A novel secreted protein involved in the regulation of bone density. Cell 89: 309-319, 1997.

22. Kazafeos K: Incretin effect: GLP-1, GIP, DPP4. Diabetes Res Clin Pract 93 (Suppl 1): S32-S36, 2011.

23. Nuche-Berenguer B, Portal-Núñez S, Moreno P, González N, Acitores A, López-Herradón A, Esbrit P, Valverde I and Villanueva-Penacarrillo ML: Presence of a functional receptor for GLP-1 in osteoblastic cells, independent of the cAMP-linked GLP-1 receptor. J Cell Physiol 225: 585-592, 2010.

24. Nuche-Berenguer B, Lozano D, Gutierrez-Rojas I, Moreno P, Marinoso ML, Esbrit P and Villanueva-Penacarrillo ML: GLP-1 and exendin-4 can reverse hyperlipidic-related osteopenia. J Endocrinol 209: 203-210, 2011.

25. Nuche-Berenguer B, Moreno P, Portal-Nuñez S, Dapía S, Esbrit P and Villanueva-Penacarrillo ML: Exendin-4 exerts osteogenic actions in insulin-resistant and type 2 diabetic states. Regul Pept 159: 61-66, 2010

26. Nuche-Berenguer B, Moreno P, Esbrit P, Dapia S, Caeiro JR, Cancelas J, Haro-Mora JJ and Villanueva-Penacarrillo ML: Effect of GLP-1 treatment on bone turnover in normal, type 2 diabetic and insulin-resistant states. Calcif Tissue Int 84: 453-461, 2009.

27. Tantikanlayaporn D, Wichit P, Weerachayaphorn J, Chairoungdua A, Chuncharunee A, Suksamrarn A and Piyachaturawat P: Bone sparing effect of a novel phytoestrogen diarylheptanoid from Curcuma comosa Roxb. in ovariectomized rats. PLoS One 8: e78739, 2013.

28. Takano-Yamamoto T and Rodan GA: Direct effects of 17 beta-estradiol on trabecular bone in ovariectomized rats. Proc Natl Acad Sci USA 87: 2172-2176, 1990.

29. Yamada C, Yamada Y, Tsukiyama K, Yamada K, Udagawa N, Takahashi N, Tanaka K, Drucker DJ, Seino Y and Inagaki N: The murine glucagon-like peptide-1 receptor is essential for control of bone resorption. Endocrinology 149: 574-579, 2008.

30. Peng J, Hui K, Hao C, Peng Z, Gao QX, Jin Q, Lei G, Min J, Qi Z, Bo C, et al: Low bone turnover and reduced angiogenesis in streptozotocin-induced osteoporotic mice. Connect Tissue Res 57: 277-289, 2016.

31. Yu SG, Zhang CJ, Xu XE, Sun JH, Zhang L and Yu PF: Ursolic acid derivative ameliorates streptozotocin-induced diabestic bone deleterious effects in mice. Int J Clin Exp Pathol 8: 3681-3690, 2015.

32. Ma B, Zhang Q, Wu D, Wang YL, Hu YY, Cheng YP, Yang ZD, Zheng YY and Ying HJ: Strontium fructose 1,6-diphosphate prevents bone loss in a rat model of postmenopausal osteoporosis via the OPG/RANKL/RANK pathway. Acta Pharmacol Sin 33: 479-489, 2012.

33. Sato T, Watanabe K, Masuhara M, Hada N and Hakeda Y: Production of IL-7 is increased in ovariectomized mice, but not RANKL mRNA expression by osteoblasts/stromal cells in bone and IL-7 enhances generation of osteoclast precursors in vitro. J Bone Miner Metab 25: 19-27, 2007. 